\title{
Abschirmungseffekte und andere Störungen
}

Hardy Zissel

Im Umgang mit RFID kommt es hin und wieder zu Situationen, in denen bestimmte Transponder nicht identifizierbar sind. Für derartige Effekte hat sich der Begriff »Abschirmung« im Sprachgebrauch etabliert.

In diesem Beitrag werden einige dieser zur Abschwächung des Magnetfeldes führender Effekte und der Begriff »Abschirmung « in Bezug auf den Faradayschen Käfig aus physikalischer Sicht betrachtet. Über das Verständnis dieser Effekte sollen dem Leser auch Möglichkeiten zur nachhaltigen Begegnung in der Praxis gegeben werden. Betrachtet werden hier HF-RFID-Systeme, die auf der Basis von rein magnetischer Kopplung arbeiten.

\section{Abschirmung physikalisch}

Mit dem Phänomen der Abschirmung, wie sie heute in der Regel von Technikern verstanden wird, hat sich bereits der englische Physiker Michael Faraday (1791-1867) befasst. Sie beruht auf dem Effekt, dass in der geschlossenen elektrisch leitenden Hülle einer Box durch zeitlich veränderliche Magnetfelder Wirbelströme entstehen, die ihrerseits Magnetfelder ausbilden, die den ursprünglichen Feldern entgegenwirken. Dadurch bleibt das innere der Box feldfrei. Da der Effekt auf Wirbelströmen basiert wird klar, dass er umso mehr wirkt, je schneller die zeitliche Varianz des Feldes erfolgt. Anders herum bedeutet das, dass Magnetfelder, die zeitlich konstant sind oder sich nur sehr langsam verändern, nicht von der elektrisch leitenden Hülle abgeschirmt werden.

Anders ist das allerdings bei magnetisch leitfähigen Materialien wie z.B. eisenhaltige Stoffe. Hierbei wird der Magnetfluss im Material entlang geleitet und somit aus dem Zentrum der Box »gezogen«. Wie weit dieser Effekt ausgeprägt ist, hängt von der magnetischen Leitfähigkeit und anderen magnetischen Eigenschaften $(\rightarrow$ Hystereseverluste) des Materials ab.

Wird das Gebilde der geschlossenen Box aus leitendem Material unter der Einwirkung von elektromagnetischen Wellen betrachtet, sieht die Wirkung noch drastischer aus. Das Innere der Box ist hier feldfrei sofern die Box für die betrachtete Frequenz tatsächlich dicht ist. Das ist immer dann der Fall, wenn sie keine Durchbrüche enthält, deren Abmessungen größer als ein Viertel der Wellenlänge sind. Dabei ist allerdings die Tiefe des Durchbruchs, also die Materialdicke, noch für die Dämpfung relevant. Da das Viertel der Wellenlänge bei HF etwa 5,5 m beträgt, ist dieses Phänomen allerdings erst im UHF Bereich relevant. Hier liegt das Viertel der Wellenlänge in der Größenordnung von 5,5 cm.

Es wird deutlich, dass Abschirmungseffekte stets mit einer Box zu tun haben, deren Inneres betrachtet wird. Diesen Aufbau findet man in der Bibliothekswelt nur in Ausnahmefällen. Dennoch sind Abschwächungseffekte oft anzutreffen. Einige werden im Folgenden behandelt. 


\section{Abschwächungseffekte aus der Praxis}

Aus der Voraussetzung zur Identifizierung von RFID-Transpondern kann leicht ableitet werden, an welchen Stellen im Prozess Störungen greifen und wirksam werden können. Zur reproduzierbaren Bestimmung der Robustheit z.B. einer Gate-Antenne gegen derartige Beeinflussungen, sind vereinheitlichte Beschreibungs- und Messverfahren in der VDI-Richtlinie 4478-1 definiert [VDI 4487-1].

Um einen Transponder zu identifizieren sind zwei wesentliche Bedingungen notwendig.

1. Der Transponder-Chip muss mit ausreichend Energie versorgt sein um arbeiten (Signale auswerten und Signale versenden) zu können.

2. Die Kommunikation zwischen Lesegerät (RFID-Reader) und Transponder muss störungsfrei verlaufen.

Die zweite Bedingung kann nur erfüllt sein, wenn die erste bereits erfüllt ist. Auf sie wird zuerst eingegangen.

\section{Die Kommunikation}

Um nicht zu tief in die Details der Informationsübertragung mittels Magnetfelder einzusteigen wird hier nur davon ausgegangen, dass die zu übertragenden Informationen in Einsen und Nullen zerlegt (codiert) übertragen werden. So gibt der Reader einen Befehl an die im Feld befindlichen Transponder, ihre eindeutige Nummer mitzuteilen. Anschließend antwortet der erste Transponder mit dieser Nummer und danach schließen sich sequentiell die nächsten im Feld befindlichen Transponder an.

Damit nun die jeweilige »Gegenstelle«, also zunächst die Transponder und dann der Reader die gesendeten Informationen eindeutig verstehen können, ist es notwendig, dass die gesendeten Einsen und Nullen in der vom Sender gewünschten Reihenfolge vom Empfänger verstanden werden. Deshalb gibt es innerhalb des Systems Reader - Transponder eindeutige Regeln, die festlegen, wer wann senden darf. Ein Transponder sendet stets nur nach Aufforderung durch den Reader. Der Reader steuert den Kommunikationsprozess.

Wenn der Reader die im Feld befindlichen Transponder zum Übertragen ihrer eindeutigen Nummer (UID) auffordert, ist er der einzige Sender. Durch über diese Betrachtungen hinausgehende Algorithmen ist sichergestellt, dass die Transponder geordnet sequentiell antworten. Bei dieser Antwort ist dann auch jeder einzelne Transponder der einzige Sender.

Wenn man sich vorstellt, dass der Inhalt der zu übertragenden Information von der richtigen Reihenfolge von Einsen und Nullen abhängt, kann man leicht nachvollziehen, dass es zu Missverständnissen kommt, wenn auch nur eine einzige Eins oder Null »verloren geht«, also nicht verstanden wird. Für diesen Fall sind Algorithmen zur Plausibilitätsprüfung in Reader und Transponder implementiert. Nur wenn nach Ablauf einer Prozedur zur Plausibilitätsprüfung festgestellt wird, dass die empfangene Information gültig ist, wird sie interpretiert und verarbeitet.

Dies hat zur Folge, dass die jeweilige Gegenseite nicht reagiert, wenn auch nur eine Eins oder Null verloren gegangen ist. Der Anwender kann dies als Abschirmung empfinden 
und beschreibt es im Allgemeinen auch so. Die physikalisch richtige Erklärung ist allerdings meist eine andere.

Um eine Eins zu übertragen, ist genau wie für eine Null eine jeweils eindeutig definierte Signalfolge erforderlich. Diese Signalfolge besteht aus unterschiedlichen Signalen genau definierter Frequenzen. Gibt es also in der Nähe der Antennen Störer, die Frequenzen aussenden, die denen der Kommunikation sehr ähnlich sind, so bekommt der Empfänger der Information Signale mit unterschiedlichen Aussagen zur gleichen Zeit. Da er, anders als z.B. das menschliche Ohr, nicht die Richtung der Signalquellen unterscheiden kann, kann er das Nutzsignal vom Störer nicht trennen. Er kann also nicht mit Sicherheit »entscheiden «, ob er gerade eine Eins, eine Null oder nur eine Störung empfangen hat. Sollte die Entscheidung nun also falsch ausfallen, wird die empfangene Information für ungültig erklärt und es erfolgt keine Reaktion. Im Extremfall kann es mit sehr geringer Wahrscheinlichkeit auch dazu kommen, dass viele falsch interpretierte Einsen und Nullen zu einer empfangenen Information führen, die in der Plausibilitätsprüfung für gültig erkannt wird. Dadurch kann es dann zu falschen Reaktionen des Empfängers führen.

Als Sender derartiger Störsignale kommen grundsätzlich alle Systeme in Frage, die in der Lage sind, magnetische Felder mit Frequenzen im Arbeitsbereich des RFID-Systems für sehr kurze Zeit abzugeben. Es reicht schließlich aus nur eine Eins oder eine Null zu stören. Grundsätzlich kommen als störende Systeme alle Geräte in Frage, in denen elektrische Ströme fließen. Man beachte bitte, dass auch das Ein- oder Ausschalten einer Kaffeemaschine Felder im Arbeitsbereich von RFID-Systemen erzeugen kann. Derartige Felder sind allerdings von sehr kurzer Dauer. Wenn ein Transponder dabei nicht erkannt wird, so wird er gleich einige Millisekunden später bei der nächsten Abfrage erkannt. Zu einer nachhaltigen Störung kann es allerdings kommen wenn elektrische Geräte zyklisch derartige Impulse abgeben. Besonders prädestiniert sind dafür andere RFID-Geräte, deren Reichweite in das betrachtete Feld hinein reicht. Dabei ist zu bedenken, dass der Reader sehr empfindlich ist um die schwachen Signale der zu identifizierenden Transponder zu erkennen. Reicht das Feld eines anderen Readers in das betrachtete Erkennungsfeld hinein, kann es zu Störungen kommen, mit denen zunächst nicht gerechnet wird, da dieser fremde Reader keine Transponder lesen kann, die sich in der Nähe des betrachteten Readers befinden. Dennoch wird das Feld dieses störenden Readers von dem hoch empfindlichen Empfänger des betrachteten Readers »wahrgenommen «.

\section{Die Energieversorgung}

Kommen wir nun zu der ersten Bedingung, die für die Identifizierung von Transpondern erfüllt sein muss. Die Energieversorgung des Chips.

Ein elektronisches System benötigt für seine Funktion elektrische Energie. Die Transponder, die in Bibliotheken verwendet werden, verfügen über keine eigene Energieversorgung. Sie sind darauf angewiesen, dass sie in dem Moment in dem sie arbeiten sollen genügend Energie zugeführt bekommen. Diese ist nur aus dem wechselnden Magnetfeld der Reader-Antenne zu beziehen. Es induziert in der Spule des Transponders (dessen 
Antenne) eine elektrische Spannung. Diese Spannung wird im Transponder-Chip gleichgerichtet und als Versorgungsspannung für seine Baugruppen verwendet. Nebenbei wird mit dem Wechselfeld auch die Information übertragen.

Ergänzend muss erwähnt werden, dass bei HF-RFID die Antennen von Reader und Transponder Elemente von resonanten Schwingkreisen sind. Es gibt also in beiden Systeme noch kapazitive Elemente, die mit den Antennenspulen verbunden sind. Durch diesen Aufbau wird die Energieübertragung wesentlich effektiver gestaltet und es sind größere Reichweiten erzielbar.

Neben dem Gewinn an Reichweite wird durch diesen Aufbau aber auch ein Nachteil erzeugt. Der Gewinn an Reichweite entsteht nur dann, wenn die Anordnung aus Induktivität (Spule) und Kapazität (Kondensator) hinreichend genau auf der Arbeitsfrequenz resonant ist.

Es sind also für die Betrachtung der Störungsmöglichkeiten der Energieübertragung mehrere elektrische Größen zu betrachten. Letztlich ist zwar nur die induzierte Spannung im Transponder ausschlaggebend, diese ist aber maßgeblich von verschiedenen anderen Dingen bestimmt.

Die Antenne des Readers muss die erforderliche magnetische Feldstäke generieren. Das geschieht, sobald durch sie ein hinreichender elektrischer Strom fließt. Dieser fließt, wenn die Ausgangsleistung des Readers groß genug ist und der Resonanzpunkt des Systems »Antenne - Kondensator « hinreichend in der Nähe der Arbeitsfrequenz liegt.

Zusätzlich wird deutlich, dass sich eine nicht so gute Resonanz mit einer höheren Reader-Leistung kompensieren lässt. Es ist also für die Betrachtung der Feldstärke des ReaderFeldes nicht ausreichend, allein die Leistung des Readers zu betrachten.

Die Antenne des Transponders muss hinreichend gut von dem Magnetfluss der ReaderAntenne durchflossen sein. Das ist der Fall, wenn die mechanische Anordnung der beiden Antennen (Reader und Transponder) zueinander optimal und der Abstand nicht zu groß ist. Die meiste elektrische Energie erhält der RFID-Chip wenn die Resonanzfrequenz der Transponder-Antenne (System aus Antennenspule und Kondensator) optimal auf der Arbeitsfrequenz abgestimmt ist.

Diese Betrachtung zeigt nicht nur die Wirkungskette auf sondern stellt auch die Punkte heraus die, bedingt durch äußere Einflüsse, Veränderungen unterliegen.

\section{Die resonante Reader-Antenne}

Die Reader-Antenne ist ein, je nach Hersteller und Antennentyp, mehr oder weniger resonantes System, was fest installiert und dabei an seine Umgebung angepasst ist. Das bedeutet, dass der Resonanzpunkt optimal auf die Arbeitsfrequenz abstimmbar ist. Da dieser Vorteil im Interesse einer optimalen Funktion auch von den Systemhäusern genutzt wird, sind die elektrischen und dielektrischen Einflüsse im unmittelbaren Umfeld der Antenne in der Bestimmung des Resonanzpunktes berücksichtigt. Wird nun 
im Betrieb das Umfeld der Antenne verändert, z.B. durch eine Person, die sich direkt in der Nähe der Antenne aufhält oder durch ein neues Möbelstück oder eine Pflanze, die nach dem Abgleich (Anpassen der Antenne an ihr Umfeld) unmittelbar an der Antenne platziert wird, wird ihr Resonanzpunkt verschoben. Das bedeutet, dass die Antenne an Empfindlichkeit verliert. Das wiederum kann dazu führen, dass Transponder von ihr schlechter erfasst werden können. Dieser Effekt des Empfindlichkeitsabfalls wird in der Praxis von Laien oft fälschlicher Weise als Abschirmung bezeichnet. Schließlich sieht es so aus, als ob eine Person, die zwischen Gate-Antenne und Transponder gelangt verhindert, dass das Magnetfeld seine Gegenstelle erreicht.

\section{Die resonante Transponder-Antenne}

Das gleiche trifft auf die Transponder-Antenne zu. Ihr Resonanzpunkt verschiebt sich, wenn ihr elektrisches oder dielektrisches Umfeld verändert wird. Das führt meist dazu, dass ihre Empfindlichkeit negativ beeinflusst wird. Veränderungen sind das Einkleben in Medien mit nicht vorhergesehenen Materialien, wie z.B. Metallanteile oder massive Kunststoffeinbände. Die Annäherung eines Transponders an einen anderen führt gleichfalls zur Verschiebung des Resonanzpunktes beider Antennen.

\section{Metallische Störer}

Es gibt noch einen Effekt, der fälschlicherweise in der Umgangssprache mit Abschirmung bezeichnet wird. Hierbei handelt es sich um die Leistungsreduzierung von Antennen bei der Annäherung an metallische Gegenstände. Tatsächlich wird hierbei zum einen, wie eben beschrieben, der Resonanzpunkt verschoben und somit die Empfindlichkeit reduziert. Dieser Effekt ist allerdings korrigierbar. Zum anderen findet aber auch eine Umleitung des Magnetflusses durch dieses Metall statt. Wenn es sich um ferromagnetische Werkstoffe, also um eisenhaltige Materialien, handelt, wird der Magnetfluss in diesem Material besser geleitet als in der Umgebungsluft. Dadurch wird die im Erkennungsfeld der Antenne benötigte Energie »umgeleitet « und verliert an der wichtigen Stelle an Wirkung. Bei Metallen im Allgemeinen kommt es zusätzlich auf Grund der elektrischen Leitfähigkeit im Material zur Indizierung eines Stromflusses. Dieser Stromfluss erzeugt wieder ein Magnetfeld welches wieder einen Stromfluss indiziert. Das setzt sich fort. Dieser Effekt wird als Wirbelstrom bezeichnet. Er führt zur Umwandlung der magnetischen Energie in Wärmeenergie. Es wird also Energie aus dem Wirkungsfeld der Antenne »vernichtet«. Wie bei der Gate-Antenne tritt dieser Effekt auch im Umfeld der Transponder-Antenne auf. Da hier die Energiemenge bedeutend geringer ist, reicht bereits eine dünne Aluminiumfolie aus um die Arbeit eines Transponders unmöglich zu machen. Auch dies ist im physikalischen Sinne keine Abschirmung.

\section{Der Gewinn für die Praxis}

Wobei helfen uns diese Gedanken nun in der Praxis? Es ist nicht nur wichtig im Interesse der Verständigung treffende Begriffe zu verwenden sondern das Wissen um die 
Hintergründe dieser Effekte hilft bei Überlegungen zur Verbesserung der Funktion von RFID-Systemen.

Es gibt behebbare und nicht behebbare Effekte. Die Einteilung ist weitestgehend klar. Effekte, die zur Verschiebung des Resonanzpunktes führen sind meist korrigierbar. Man benötigt »nur « die entsprechende »Stellschraube« dafür. Diese ist bei Gate-Antennen meist sogar in Form einer veränderlichen Kapazität direkt vorhanden. Bei Transpondern ist das schwieriger. Ihre Eigenschaften werden bei der Herstellung weitestgehend festgelegt. Die Bibliothek hat hier keinen wirtschaftlich vertretbaren Einfluss. Wenn allerdings den Herstellern von Gate-Antennen und Transpondern Möglichkeiten zur Verfügung stünden, die Resonanz nur gering nutzen zu müssen, also die Resonanzkurve flach (die Kreisgüte gering) zu halten, würden sich Verschiebungen dieser Resonanzkurve weniger bemerkbar machen und die Produkte wären vielseitiger verwendbar. Derartige Möglichkeiten liegen in der Erhöhung der Empfindlichkeit der Empfänger der Gate-Antennen und der Transponder-Chips.

Zu den schwer beeinflussbaren Effekten zählt die Umleitung des Magnetflusses und die Wirbelstrombildung in Metallen. Das Wissen darum hilft bei der Planung von GateAnlagen und bei der Platzierung von Transpondern an metallhaltigen Medien. Begegnen kann man diesem Effekt nur durch aufwendige Führung des Magnetflusses und in begrenztem Maße durch die Erhöhung der Leistung der Antenne. Der Leistungserhöhung sind allerdings zwei Grenzen gesetzt. Eine Grenze ist eine physikalische. Es lässt sich zwar die Leistung des Senders eines Gates erhöhen um die benötigte Energie den Transpondern zur Verfügung zu stellen. Allerdings wird durch den Effekt im Metall auch die Leistung geschwächt, die vom Transponder beim Empfänger des Gates ankommt. Wenn der Transponder nicht mehr verstanden wird, bringt eine weitere Leistungserhöhung im Gate keinen Effekt. Eine andere Grenze ist der Leistungserhöhung durch die Norm EN300330 gesetzt. Diese Norm geht davon aus, dass ein HF-RFID-Gate keine reguläre Funkanlage ist. Aus diesem Grundgedanken ergibt sich eine starke Beschränkung der abgestrahlten Leistung bei der Arbeitsfrequenz von 13,56 MHz.1

Die hier im Einzelnen betrachteten Effekte zeichnen sich durch die gleiche vom Anwender spürbare Wirkung aus - die Nichterkennung von Transpondern - obwohl sich aus technischer Sicht unterschiedliche Ursachen und Wirkungsmechanismen dahinter verbergen. Durch eine präzise differenzierte Betrachtung kann es in der Praxis gelingen eine Vielzahl von Beeinträchtigungen zu Unterbinden oder zu minimieren und die Funktion von RFID-Systemen angenehmer zu gestalten.

\section{Quelle}

[VDI 4478-1] »Testverfahren zur Vereinheitlichung Leistungsbestimmung von RFID-Gates für den Einsatz in Bibliotheken«

1 Nur spezielle Antennenkonstruktionen generieren einen starken Magnetfluss im Erkennungsfeld bei gleichzeitig geringer Störabstrahlung innerhalb des Normwertes. 